\title{
THE ALGEBRAIC INDEPENDENCE OF CERTAIN LIOUVILLE CONTINUED FRACTIONS
}

\author{
WILLIAM W. ADAMS ${ }^{1}$
}

\begin{abstract}
This work uses some simple Liouville type arguments to extend some recent work of Bundschuh and of Laohakosol and Ubolsri on algebraic independence. The results are stronger and are not restricted to just two numbers. We then use the results to give a new and simple proof of Bundschuh's result concerning the algebraic independence of certain numbers whose $g$-adic and continued fraction expansions are both known.
\end{abstract}

1. Introduction. In this paper we generalize and strengthen the results of Laohakosol and Ubolsri [11]. In that paper they prove by the method of $[1,9,14]$ the algebraic independence of certain pairs of "Liouville" continued fractions. Here a similar result is given for any number of such numbers. Also, the hypothesis is weakened allowing a restriction in their application to be removed. The work here also generalizes some of the work of Bundschuh in [5] from two numbers to an arbitrary number of numbers. The application given in [11] was first proved by Bundschuh in [4] in more generality by a complicated method following Durand [8]. The full result can be proved here by the current simpler method.

We fix the following notation. Let $\alpha_{1}, \ldots, \alpha_{n}$ be $n$ real numbers with continued fraction expansions

$$
\alpha_{j}=\left[a_{0 j} ; a_{1 j}, a_{2 j}, \ldots\right] .
$$

(See [10], for example, for facts concerning continued fractions.) Denote the convergents of $\alpha_{j}$ by $p_{N_{j}} / q_{N_{j}}(n=0,1,2, \ldots, 1 \leqslant j \leqslant n)$.

THEOREM 1. Suppose we have an $r>1$ and a function $f(i)$ for $i=1,2, \ldots$ with $f(i) \rightarrow \infty(i \rightarrow \infty)$ and a subsequence of the positive integers $N_{1}<N_{2}<\cdots$ such that, for all $i=1,2, \ldots$,

$$
\begin{gathered}
a_{N_{i}+1, j} \geqslant q_{N_{1} 1}^{f(i)} \quad(j=1,2, \ldots, n), \\
q_{N, j-1} \geqslant r^{f(i)} q_{N_{j}} \quad\left(j=2,3, \ldots, n, N=N_{i}, N_{i}+1\right) .
\end{gathered}
$$

Then $\alpha_{1}, \ldots, \alpha_{n}$ are algebraically independent.

Received by the editors November 30, 1984 and, in revised form, January 31, 1985.

1980 Mathematics Subject Classification. Primary 10F37, 10F20.

Key words and phrases. Algebraic independence, continued fractions, Liouville numbers.

${ }^{1}$ Partially supported by NSF Grant MCS83-01431. 
To see that this result generalizes the results of $[5,11]$ we note

COROllaRY 2. Suppose there are constants $\tau, r>1$ and a function $g(i)$ for $i=1,2, \ldots$ with $g(i) \rightarrow \infty(i \rightarrow \infty)$ and a subsequence of the positive integers $N_{1}<N_{2}<\cdots$ such that, for all $N=1,2, \ldots$ and $j=2, \ldots, n$, we have

$$
\begin{aligned}
& a_{N+1,1} \geqslant a_{N 1}^{\tau}, \\
& a_{N, j-1} \geqslant r a_{N j}, \\
& a_{N_{i}+1, j} \geqslant a_{N_{i} 1}^{g(i)} .
\end{aligned}
$$

Then $\alpha_{1}, \ldots, \alpha_{n}$ are algebraically independent.

As an application we prove a result which first appeared in [4].

COROLlaRY 3. For any integer $g \geqslant 2$ and irrational number $\beta$ set

$$
S_{g}(\beta)=(g-1) \sum_{\nu=1}^{\infty} g^{-[\nu \beta]} .
$$

Then, if $\beta$ has unbounded partial quotients and $g_{1}, \ldots, g_{n}$ are distinct integers $\geqslant 2$, we have that $S_{g_{1}}(\beta), \ldots, S_{g_{n}}(\beta)$ are algebraically independent.

2. A general Liouville type algebraic independence criteria. In this section we axiomatize the proofs given in $[9,11,14]$.

THEOREM 4. Let $\alpha_{1}, \ldots, \alpha_{n}$ be $n$ real numbers. Assume that we are given integers $p_{N j}$, $q_{N_{j}}(N=1,2, \ldots$ and $1 \leqslant j \leqslant n)$ with $q_{N_{j}} \rightarrow \infty(N \rightarrow \infty)$. Assume that, for $j=$ $2,3, \ldots, n$,

$$
\lim _{N \rightarrow \infty}\left|\alpha_{j-1}-\frac{p_{N, j-1}}{q_{N, j-1}}\right| /\left|\alpha_{j}-\frac{p_{N j}}{q_{N j}}\right|=0 .
$$

Further assume that for each $j=1,2, \ldots, n$ and all positive integers $D$ there is an $N_{0}=N_{0}(D)$ such that, for all $N>N_{0}$,

$$
0<\left|\alpha_{j}-p_{N_{j}} / q_{N_{j}}\right|<1 /\left(q_{N 1} \cdots q_{N_{j}}\right)^{D} \text {. }
$$

Then $\alpha_{1}, \ldots, \alpha_{n}$ are algebraically independent.

Proof. We show by induction on $j=1,2, \ldots, n$ that $\alpha_{1}, \ldots, \alpha_{j}$ are algebraically independent. For $j=1$ we note that (7) implies that $\alpha_{1}$ is a Liouville number (see [12]) and thus is transcendental. So we assume $j>1$. If the result were false, then there would be a nonzero polynomial $f\left(x_{1}, \ldots, x_{j}\right)$ with integer coefficients of minimal total degree such that $f\left(\alpha_{1}, \ldots, \alpha_{j}\right)=0$. Expand $f$ in a Taylor series about $\alpha_{1}, \ldots, \alpha_{j}$

$$
f\left(x_{1}, \ldots, x_{j}\right)=\sum c_{(\nu)}\left(x_{1}-\alpha_{1}\right)^{\nu_{1}} \cdots\left(x_{j}-\alpha_{j}\right)^{\nu_{j}},
$$

where $(\nu)=\left(\nu_{1}, \ldots, \nu_{j}\right)$. For $i=1, \ldots, j$, set $C_{i}=c_{(\nu)}$ where $\nu$ has a 1 in the $i$ th coordinate and a zero everywhere else. We have

$$
C_{j}=\left(\partial / \partial x_{j}\right) f\left(\alpha_{1}, \ldots, \alpha_{j}\right) .
$$


Then $C_{j}=0$ implies $\left(\partial / \partial x_{j}\right) f\left(x_{1}, \ldots, x_{j}\right) \equiv 0$ or else there would be a nonzero polynomial of smaller degree than $f$ vanishing at $\alpha_{1}, \ldots, \alpha_{j}$. But then $x_{j}$ did not occur in the polynomial $f$ and we obtain that $\alpha_{1}, \ldots, \alpha_{j-1}$ are algebraically dependent, violating our induction assumption. Thus $C_{j} \neq 0$. Setting

$$
\delta_{i}(N)=p_{N i} / q_{N i}-\alpha_{i} \quad(1 \leqslant i \leqslant j)
$$

we obtain

$$
\begin{aligned}
& f\left(\frac{p_{N 1}}{q_{N 1}}, \ldots, \frac{p_{N j}}{q_{N j}}\right) \\
& \quad=\delta_{j}(N)\left[\left(C_{1} \frac{\delta_{1}(N)}{\delta_{j}(N)}+\cdots+C_{j-1} \frac{\delta_{j-1}(N)}{\delta_{j}(N)}+C_{j}\right)+O\left(\left|\delta_{j}(N)\right|\right)\right]
\end{aligned}
$$

$\left(\left|\delta_{j}(N)\right|\right.$ is the largest by (6)). The expression inside the square bracket is not zero for $N$ large by (6) and the fact that $C_{j} \neq 0$. Thus $\delta_{j}(N) \neq 0$ implies $f\left(p_{N 1} / q_{N 1}, \ldots, p_{N j} / q_{N j}\right) \neq 0$ for $N$ large. We then easily derive (see [1, Proposition 3]) that if $D_{1}, \ldots, D_{j}$ denotes the degree of $f$ in $x_{1}, \ldots, x_{j}$, respectively, there is a constant $C=C\left(\alpha_{1}, \ldots, \alpha_{j}, f\right)$ such that, for all $N$ large,

$$
\frac{1}{q_{N 1}^{D_{1}} \cdots q_{N_{j}}^{D_{j}}} \leqslant\left|f\left(\frac{p_{N 1}}{q_{N 1}}, \ldots, \frac{p_{N_{j}}}{q_{N_{j}}}\right)\right| \leqslant C\left|\alpha_{j}-\frac{p_{N_{j}}}{q_{N j}}\right|
$$

which violates (7).

As was first pointed out to me by David Masser, this result gives a trivial proof of the results in [1], and indeed proves

COROLlaRY 5. Let $k_{\nu}(\nu=1,2, \ldots)$ be a strictly increasing sequence of positive integers such that $\lim \sup _{N \rightarrow \infty} k_{N+1} / k_{N}=\infty$. Let $g_{1}, \ldots, g_{n}$ be distinct integers $\geqslant 2$ and set $\alpha_{j}=\sum_{\nu=1}^{\infty} g_{j}^{-k_{\nu}}$. Then $\alpha_{1}, \ldots, \alpha_{n}$ are algebraically independent.

(This result occurs in [13].)

Proof. We may assume $g_{1}>g_{2}>\cdots>g_{n}$. Set $p_{N j} / q_{N j}=\sum_{\nu=1}^{N} g_{j}^{-k_{\nu}}$. We have

$$
q_{N j}=g_{j}^{k_{N}} \text { and } 1 / g_{j}^{k_{N+1}}<\left|\alpha_{j}-p_{N j} / q_{N j}\right|<2 / g_{j}^{k_{N+1}} \text {. }
$$

Then (7) is implied by

$$
2 / g_{j}^{k_{N+1}} \leqslant 1 /\left(g_{1}^{k_{N}} \cdots g_{j}^{k_{N}}\right)^{D}
$$

which is clear for a subsequence if $N$ tending to infinity. Moreover, (6) is clear since the relevant expression is bounded above by $\left(2 / g_{j-1}^{k_{N+1}}\right) g_{j}^{k_{N+1}}$ which tends to zero.

3. Proof of the results on continued fractions. To prove Theorem 1 we verify the hypothesis of Theorem 4 with the $N$ of Theorem 4 replaced by the $N_{i}$ of Theorem 1 . To prove (6) we note that the usual estimates from continued fractions and (2) imply

$$
\left|\alpha_{j-1}-\frac{p_{N_{i}, j-1}}{q_{N_{i}, j-1}}\right| /\left|\alpha_{j}-\frac{p_{N_{i} j}}{q_{N_{i} j}}\right| \leqslant \frac{2 q_{N_{i}+1, j} q_{N_{i}, j}}{q_{N_{i}+1, j-1} q_{N_{i}, j-1}} \leqslant 2 r^{-2 f(i)}
$$


which tends to zero by hypothesis. To verify (7) we use

$$
\left|\alpha_{j}-\frac{p_{N_{j}}}{q_{N_{j}}}\right| \leqslant 1 / a_{N+1, j} q_{N_{j}}^{2}
$$

and see that it suffices to prove that, for $i>i_{0}(D)$,

$$
a_{N_{i}+1, j} \geqslant\left(q_{N_{1} 1} \cdots q_{N_{i}}\right)^{D} \text {. }
$$

From (2) we have

$$
\left(q_{N_{i} 1} \cdots q_{N_{i} j}\right)^{D} \leqslant q_{N_{i} 1}^{j D}
$$

and so (8) follows immediately from (1).

Corollary 2 may be derived from Theorem 1 by showing that (3), (4) and (5) implies (1) and (2). From Lemma 3 of [5] we have from (4) that $q_{N, j-1} \geqslant r^{N / 2} q_{N j}$ for all $N=1,2, \ldots$ and $j=2, \ldots, n$. Thus (2) is valid for any $f(i) \leqslant N_{i} / 2$. To verify (1) we will show that (3) implies there is a constant $C>0$ such that, for all $N=1,2, \ldots$,

$$
q_{N 1} \leqslant C a_{N 1}^{\tau /(\tau-1)}
$$

Assuming this and using (5) we see

$$
a_{N_{i}+1, j} \geqslant a_{N_{i} 1}^{g(i)} \geqslant\left(C^{-1} q_{N_{i} 1}\right)^{((\tau-1) / \tau) g(i)}
$$

which implies (1) for, say, $f(i) \leqslant \frac{1}{2}((\tau-1) / \tau) g(i)$, and $i$ large. We may take a new sequence by starting with $i$ large and $f(i) \leqslant N_{i} / 2, \frac{1}{2}((\tau-1) / \tau) g(i)$.

It remains to prove (9). We have from (3) that, for $\nu=0,1,2, \ldots, N-1, a_{N 1} \geqslant$ $a_{N-\nu, 1}^{\tau^{\nu}}$, and thus

$$
\begin{aligned}
q_{N 1} & \leqslant \prod_{\nu=1}^{N}\left(a_{N-\nu+1,1}+1\right) \\
& \leqslant \prod_{\nu=1}^{N}\left(a_{N 1}^{1 / \tau^{\nu-1}}+1\right)=\prod_{\nu=1}^{N} a_{N 1}^{1 / \tau^{\nu-1}}\left(1+a_{N 1}^{-1 / \tau^{\nu-1}}\right) .
\end{aligned}
$$

Now,

$$
\prod_{\nu=1}^{N} a_{N 1}^{1 / \tau^{\nu-1}}=a_{N^{\prime}-1 / \tau^{\nu-1}}^{\sum^{N}}=a_{N 1}^{\left.\left(1-1 / \tau^{N}\right) / 1-1 / \tau\right)} \leqslant a_{N 1}^{\tau /(\tau-1)} .
$$

Also $a_{11} \geqslant 2$ (from (4)) and thus $a_{N 1} \geqslant 2^{\tau^{N-1}}$ (from (3)) and so

$$
\prod_{\nu=1}^{N}\left(1+a_{N 1}^{-1 / \tau^{\nu-1}}\right) \leqslant \prod_{\nu=1}^{N}\left(1+2^{-\tau^{N-\nu}}\right) \leqslant C
$$

for some constant $C$. This proves (9).

4. Proof of Corollary 3. Corollary 3 will be deduced from Theorem 1. We may assume that $g_{1}>g_{2}>\cdots>g_{n}$. Set $\alpha_{j}=S_{g_{j}}(\beta)$. Let $\beta^{-1}$ have as its continued fraction expansion

$$
\beta^{-1}=\left[b_{0} ; b_{1}, b_{2}, \ldots\right]
$$

and convergents $P_{N} / Q_{N}(N=0,1,2 \ldots)$. Then from [2] (see also $\left.[3,4,6]\right)$ we have

$$
a_{0 j}=b_{0} g_{j}, \quad a_{N j}=\left(g_{j j}^{Q_{N}}-g_{j}^{Q_{N-2}}\right) /\left(g_{j}^{Q_{N-1}}-1\right)
$$


and

$$
q_{N j}=\left(g^{Q_{N}}-1\right) /\left(g_{j}-1\right) .
$$

It easily follows from (10) that $a_{N+1, j} \geqslant g_{j}^{\left(b_{N+1}-1\right) Q_{N}}$ and from (11) that $q_{N 1} \leqslant g_{1}^{Q_{N}}$, and thus (1) follows since $\beta$ has unbounded partial quotients. To prove (2) we simply note that (11) implies, for each $j, g_{j}^{Q_{N}-1} \leqslant q_{N_{j}} \leqslant g_{j}^{Q_{N}}$ and thus, for any $N$,

$$
q_{N, j-1} \geqslant g_{j-1}^{Q_{N}-1}=g_{j-1}^{-1}\left(\frac{g_{j-1}}{g_{j}}\right)^{Q_{N}} g_{j}^{Q_{N}} \geqslant g_{j-1}^{-1}\left(\frac{g_{j-1}}{g_{j}}\right)^{Q_{N}} q_{N_{j}} .
$$

Thus (2) is valid since $g_{j}<g_{j-1}$ by assumption (the subsequence is unnecessary).

\section{REFERENCES}

1. W. W. Adams, On the algebraic independence of certain Liouville numbers, J. Pure Appl. Algebra 13 (1978), 41-47.

2. W. W. Adams and J. L. Davison, A remarkable class of continued fractions, Proc. Amer. Math. Soc. 65 (1977), 194-198.

3. P. E. Böhmer, Über die Transzendenz gewisser dyadischer Brüche, Math. Ann. 96 (1927), 367-377.

4. P. Bundschuh, Über eine Klasse reeler Transzendenter Zahlen mit explizit angebbarer g-adischer und Kettenbruch-Entwicklung, J. Reine Angew Math. 318 (1980), 110-119.

5. __ Transcendental continued fractions, J. Number Theory 18 (1984), 91-98.

6. L. V. Danilov, Some classes of transcendental numbers, Mat. Zametki 12 (1972), 149-154 (Russian); English transl., Math. Notes 12 (1972), 524-527.

7. J. L. Davison, A series and its associated continued fraction, Proc. Amer. Math. Soc. 63 (1977), 29-32.

8. A. Durand, Indépendence algébrique de nombres complexes et critère de transcendance, Compositio Math. 35 (1977), 259-267.

9. Y. Flicker, Algebraic independence by a method of Mahler, J. Austral. Math. Soc. Ser. A 27 (1979), 173-188.

10. S. Lang, Introduction to Diophantine approximations, Addison-Wesley, Reading, Mass., 1966.

11. V. Laohakosol and P. Ubolsri, Some algebraically independent continued fractions, Proc. Amer. Math. Soc. (to appear).

12. W. J. LeVeque, Topics in number theory, Addison-Wesley, Reading, Mass., 1956.

13. R. Pass, Results concerning the algebraic independence of sets of Liouville numbers, Ph. D. Thesis, Univ. of Maryland, College Park, Md., 1978.

14. I. Shiokawa, Algebraic independence of certain gap series, Arch. Math. 38 (1982), 438-442.

Department of Mathematics, University of Maryland, College Park, Maryland 20742 